\title{
ALL-OPTICAL SWITCHING IN HOT ATOMS WITH STANDING-WAVE DRIVE
}

\section{XUE MEI SU1 ${ }^{1}$, RUI ZHANG ${ }^{1}$, HUIFANG ZHANG ${ }^{2}$ and ZHONG CHANG ZHUO ${ }^{1}$}

${ }^{1}$ College of Physics

Jilin University

Changchun 130012

P. R. China

e-mail: suxm@jlu.edu.cn

2Department of Physics

Shanghai University

99 Shangda Road, Baoshan District

Shanghai 200444

P. R. China

e-mail: zhuozc@jlu.edu.cm

\begin{abstract}
We propose a scheme of all-optical switching in hot inverse Y-type four-level atoms driven by a strong standing-wave (SW). Two optical blockades are induced by quantum coherence of the strong standing-wave field at individual wavelengths of two travelling-wave (TW) probe fields. The blockade at probe field 2 can be burst when probe field 1 becomes strong and saturated absorption takes effect under keeping two-photon resonance. So the first probe field can switch on or off the second probe field all-optically with different strength in hot standing-wave-dressed atoms. In the other hand, when the second probe field is
\end{abstract}

2010 Mathematics Subject Classification: 81V80, 81V45, 81P40.

Keywords and phrases: quantum coherence, standing wave drive, optical switching.

Received May 27, 2019; Revised June 14, 2019

(C) 2019 Scientific Advances Publishers 
resonant to the respective atomic transition to create EIT (electromagnetically induced transparency), the system can do one-order temporal differentiate calculation to the first probe field with Gaussian shape. The scheme has potential applications in all-optical transistor in quantum logical circuits.

\section{Introduction}

By applying external coherent fields, atoms can undergo controllable change in their internal structures and coherent excitation [1, 2, 3]. This leads to many novel effects of quantum coherence. For example, alloptical transistors [4], one of the most important devices in quantum logical circuits, can be realized by external coupling fields in cold atoms [5]. However, cold atomic system has inherent limitation to relax atomic populations and thereby destroy quantum coherence. The hot atoms become an alternative choice for many quantum logic proposals. Therefore, dressed atoms [6, 7] without laser cooling have been investigated intensively. However, all-optical devices $[8,9]$ in hot atomic system have to suppress the broader spectrum line widths in order to reduce destroying impacts from atomic moving. A various of Doppler-free techniques have be utilized for this purpose, such as electromagnetically induced transparency (EIT) [10, 11], hole-burning [12] and coherent population oscillation [13], transferred quantum states [14], coupled atomic coherence $[15,16]$ with a standing-wave drive. Here, we propose a Doppler-free configuration using EIT and coupled coherence of a standing wave drive to realize two-color photonic blockades and further be demonstrated as an all-optical transistors. A two-photonic-blockade on two weak probe fields can be produced within the transparent windows due to coupled coherence of the standing wave field [15]. Recently, a single photonic blockade by a standing wave field have been observed in proposals of electromagnetically induced photonic bandgap [16], optical switching [17], and light storage [18]. The two-color photonic blockades are useful to make a transistor, which can be used as a switch or a logical device in quantum circuits. 


\section{Materials and Methods}

The energy-level diagram of the system is shown in Figure 1 where Figure 1(a) shows the diagram of four energy states of bare atoms; Figure 1(b) exhibits the dressed states in which the dressed splitting doublets $\mid 2 d^{+}>$and $\left|3 d^{+}>,\right| 2 d^{-}>$and $\mid 3 d^{-}>$are caused by two TW (travelling wave) components of SW field $\grave{U}_{+}$and $\grave{U}_{-}$, respectively. The $\grave{U}_{+}$dressed and $\grave{U}_{-}$dressed atoms (indicated by signs ++ and -- in Figure 1(c)) lead to two-photon electromagnetically induced transparency or absorption (EIA) dependent to intensities of probe field $\grave{U}_{p 1}$ and $\grave{U}_{p 2}$. Electromagnetically induced two-photon transparency (EITT) is resulted from atomic coherence between two respect transition paths $\mid 1>$ $-\left|2 d^{ \pm}>-\right| 4>$ and $|1>-| j 3 d^{ \pm}>\mid 4>$, respectively [7, 19]. The coupled coherence between two types of dressed hot atoms can lead to Bragg reflections off the absorption or transparent peaks [18]. The photonic blockades to the two probe fields from Bragg reflection are indicated by signs +- in dotted rectangles in Figure 1(c). The strong SW field creates two-color optically blockades [17] on propagation of two probe fields in the atoms as function of two $p$ - $n$ junctions on electronic fluxing in an electronic transistor. 


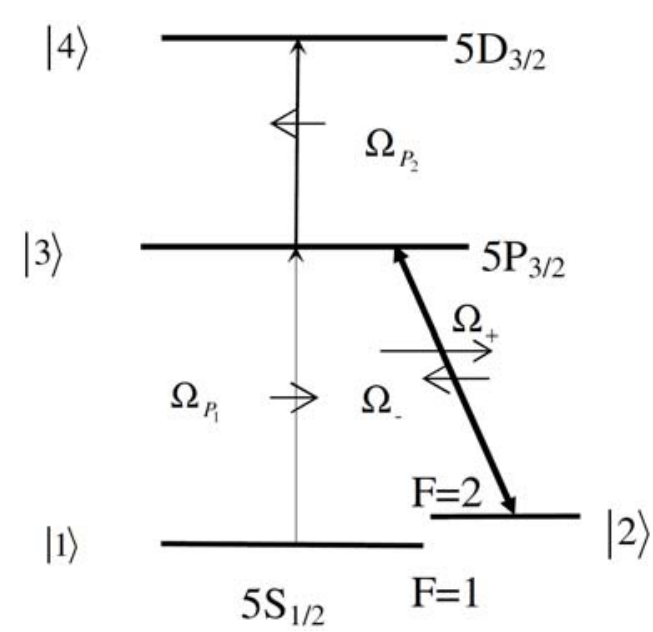

(a)

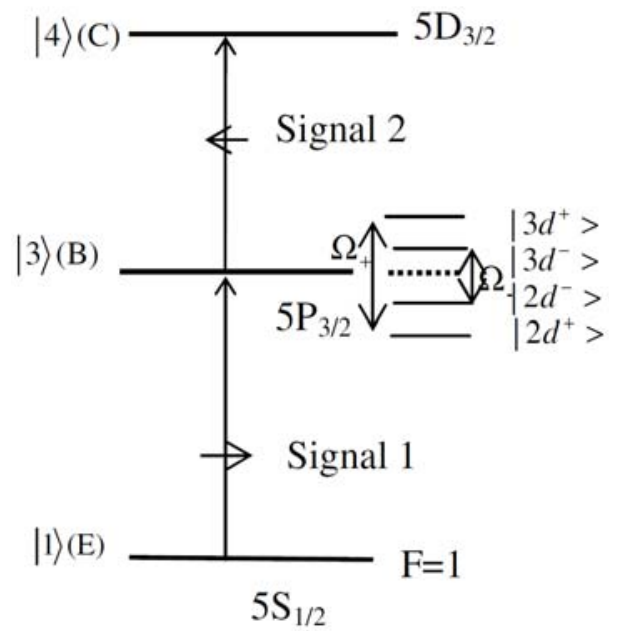

(b)

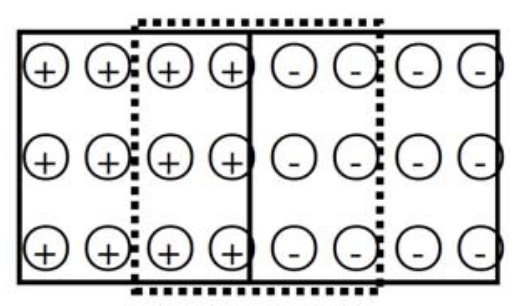

(c)

Figure 1. (a) Energy-level diagram of the four-level system in bare states; levels $|1>| 2>,, \mid 3>$, and $\mid 4>$ correspond to $\mid 5 S 1 / 2, F=1>$, $|5 S 1 / 2, F=2>| 5 P 3 / 2>$, and $\mid 5 D 3 / 2>$ states of the Rb atoms, respectively. (b) the equivalent "dressed" energy level schematic of the system in Figure 1; the amplitude modulation effects of $\Omega_{+}$and $\Omega_{-}$lead to dressed splitting doublets $\mid 2 d^{+}>$and $\left|3 d^{+}>,\right| 2 d^{-}>$and $\mid 3 d^{-}>$, respectively. (c) $\Omega_{+}$- and $\Omega_{-}$- dressed atoms are indicated by signs ++ and -- in solid rectangles. The phase modulation effect between the two types of dressed atoms results in $p-n$ junction area indicated by signs +in dotted rectangle. 
Considering the system in Figure 1(a), Hamilton of the system in interaction picture

$$
\begin{gathered}
H=-\hbar\left(\Delta_{p 1}-\Delta_{c}\right)|2><2|-\hbar \Delta_{p 1}|3><3|-\hbar\left(\Delta_{p 1}+\Delta_{p 2}\right)|4><4| \\
-\hbar \Omega_{c}|3><2|-\hbar \Omega_{p 1}|3><1|-\hbar \Omega_{p 2}|4><3|
\end{gathered}
$$

where $\Delta_{p 1}=\left(\omega_{p 1}-\omega_{31}\right), \Delta_{p 2}=\left(\omega_{p 2}-\omega_{43}\right)$, and $\Delta_{c}=\left(\omega_{c}-\omega_{32}\right)$ are frequency detunings of probe fields $\Omega_{p 1}, \Omega_{p 2}$ and standing wave field with corresponding atomic transitions; Rabi frequencies of the respective incident lasers are $\Omega_{p 1}=\left|\Omega_{1}\right| e^{-i \mathbf{k}_{p 1} \cdot r}, \Omega_{p 2}=\left|\Omega_{2}\right| e^{i \mathbf{k}_{p 2} \cdot r}, \Omega_{c}(z)=\left|\Omega_{+}\right| e^{-i k_{c} \cdot z}$ $+\left|\Omega_{-}\right| e^{i k_{c} \cdot z} ; \Omega_{1}=g_{31} E_{p 1}, \Omega_{2}=g_{43} E_{p 2}, \Omega_{ \pm}=g_{32} E_{ \pm}, 2 \hbar g_{i j}(i, j=1,2,3,4)$ are dipole matrix elements of atomic transitions, $E_{p 1, p 2, \pm}$ are slow varying field amplitudes, while $\mathbf{k}_{p 1, p 2}$ and $k_{c}$ are wave vectors of the two probe fields and standing wave field, respectively.

The density matrix equations in the hot atoms can be obtained by inserting expression (1) into the interaction equation as [20]:

$$
\left(\frac{\partial}{\partial t}+\nu \frac{\partial}{\partial z}\right) \psi=Q \psi+I
$$

where column vectors $\psi$ and $I$ are:

$\psi=\left(\rho_{11}, \rho_{12}, \rho_{13}, \rho_{14}, \rho_{21}, \rho_{22}, \rho_{23}, \rho_{24}, \rho_{31}, \rho_{32}, \rho_{33}, \rho_{34}, \rho_{41}, \rho_{42}, \rho_{43}\right)^{T}$,

$$
I=\left(\begin{array}{lllllllllllllll}
\Gamma_{41} & 0 & 0 & 0 & 0 & \Gamma_{42} & 0 & 0 & 0 & 0 & \Gamma_{43} & i \Omega_{p 2}^{*} & 0 & 0 & -i \Omega_{p 2}
\end{array}\right)^{T} .
$$

Here superscript $T$ stands for being transposed, matrix $Q$ is the same as in [15]. To make Fourier transformation to Equation (2) and expend $\Psi$ in spatial Fourier series as:

$$
\Psi(j, v)=\sum X_{j}(m, \nu) e^{i m k_{c} z} * \operatorname{phase}(j),
$$


and

$$
\operatorname{phase}(j)=\left\{\begin{array}{c}
e^{0}(j=1,6,7,10,11), \\
e^{-i\left(k_{p 1}-k_{p 2}\right) z}(j=13), \\
e^{i\left(k_{p 1}-k_{p 2}\right) z}(j=4), \\
e^{-i k_{p 1} z}(j=2,3), \\
e^{i k_{p 1} z}(j=5,9), \\
e^{-i k_{p 2} z}(j=8,12), \\
e^{i k_{p 2} z}(j=14,15) .
\end{array}\right.
$$

By substituting Equation (5) into Equation (2), we get an infinite series of solutions [20] of $X j$ and $\psi$, which are dependent to atomic velocity and stand for the slowly varying amplitudes of the densitymatrix operator in frequency domain. The absorption, transmission and reflection of the output signals in time domain are obtained by making reverse Fourier transformation for solutions of all the atomic velocity classes to Equation (2). The total atomic polarization at probe fields can be obtained by integration to all the atomic velocity under Maxwell velocities distribution.

\section{Results and Discussion}

In order to show the switching properties, we assume the wave front of probe field 1 (as the input field) has a square wave form as shown in Figure 2(a). The transmission and reflection of the output field (probe field 2) is determined by susceptibility of probe field 2 which is proportional to the matrix element $\rho_{43}(m)$. It is integrated under Maxwell velocities distribution to all responses of atoms considering the atomic motion:

$$
\rho_{43}=\sum_{m=-\infty}^{m=\infty} \int_{-\infty}^{\infty} X_{15}(m, \nu) N(\nu) d \nu e^{i m k_{c} z} \quad(m=0, \pm 1, \pm 2, \ldots),
$$


where $N(\nu)=e^{-\nu^{2} / \nu_{p}^{2}} / \nu_{p} \sqrt{\pi}$ is the Maxwell distribution, $\nu_{p}$ represents the most probable atomic speed. Because susceptibility is modulated periodically by standing wave field, we apply method of transfer matrix [21] to calculate the transmission, reflection, and absorption of the output of the signal field. Time evolution for the output signals in time domain is obtained as shown in Figures 2(b), 2(c), and 2(d), respectively. From Figure 2, we found the weak controlling field results in output signal absorbed in the SW-dressed atoms and thus working at its state off due to two optical blockades are formed. However, the output of FWM signal can be transmitted through the system since the optical blockade is burst with assist of a strong input signal and so the system working at its state on. The SW-dressed atoms take impacts on the signal pulse whose duration in order of $\mu s$, because of decaying rates of the excited levels $\mid 3>$ and $\mid 4>$ in several MHz. To switch shorter pulse, we can choose the media with the decaying rates in respect orders. 


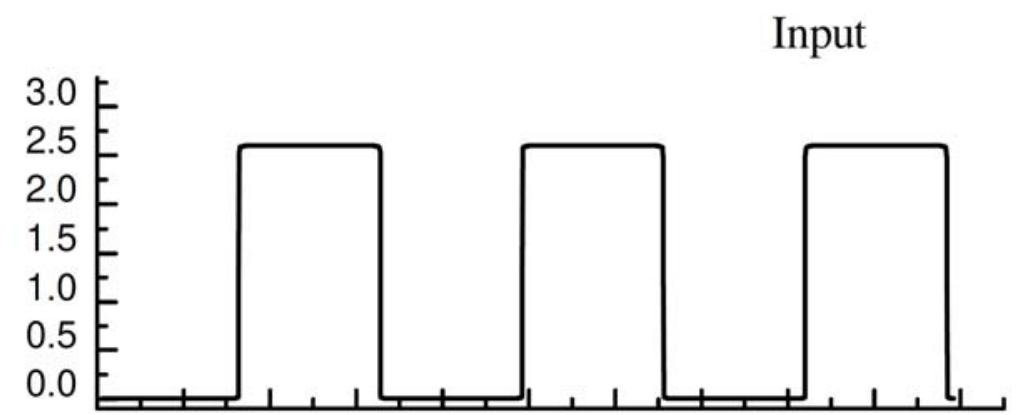

(a)

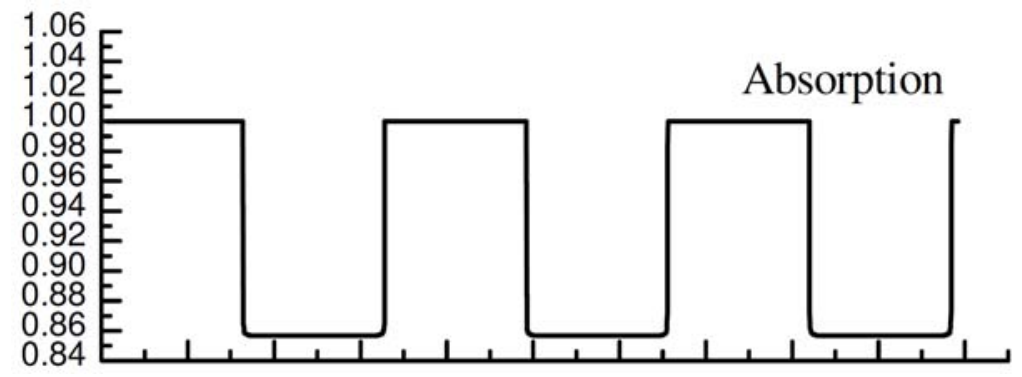

(b)

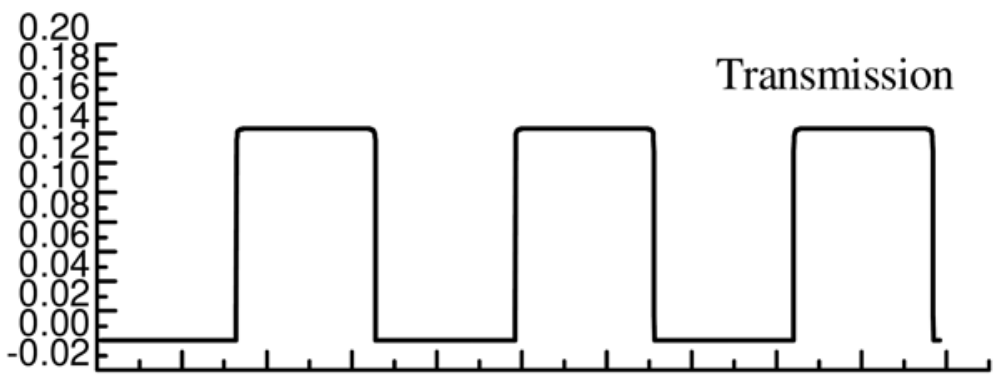

(c) 


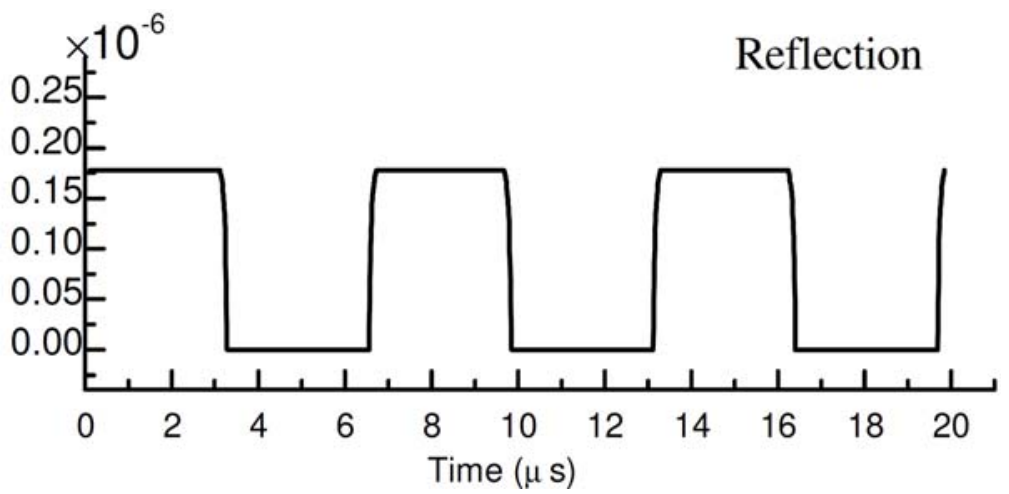

(d)

Figure 2. (a) Waveforms of the control field (probe field 1), whose Rabi frequency is in unit of spontaneous emission rate between levels $\mid 3>$ and $1>, \gamma=2 \pi \times 6.065 \mathrm{MHz}$; (b) the absorptive; (c) transmitted; and (d) reflected waveforms of the signal field (probe field 2); the used parameters are: atomic density $N=10^{11} \mathrm{~cm}^{-3}, \Omega_{ \pm}=7.5 \gamma, \Omega_{p 2}=0.026 \gamma$.

Similarly, when the system is working in EIT configuration [15] under $\Delta_{p 2}=0$, the transmission and reflection of the first probe field (as the input signal) is determined by its susceptibility in the system which is proportional to the matrix element $\rho_{31}(m)$ :

$$
\rho_{31}=\sum_{m=-\infty}^{m=\infty} \int_{-\infty}^{\infty} X_{7}(m, \nu) N(\nu) d \nu e^{i m k_{c} z} \quad(m=0, \pm 1, \pm 2, \ldots) .
$$

If the signal entering the system with a Gaussian shape as $E(t)=E_{p 0}$ $\exp \left(-t^{2} / \tau^{2}\right) \exp \left(-i \omega_{0} t\right)$ ( $\tau$ is the duration of the input pulse), the output signal through the atomic system will have a shape of the first-order differential of Gauss pulse as shown in Figure 3. The system can do differentiate calculation in a certain situation and therefore takes as a component in the quantum circuits. 


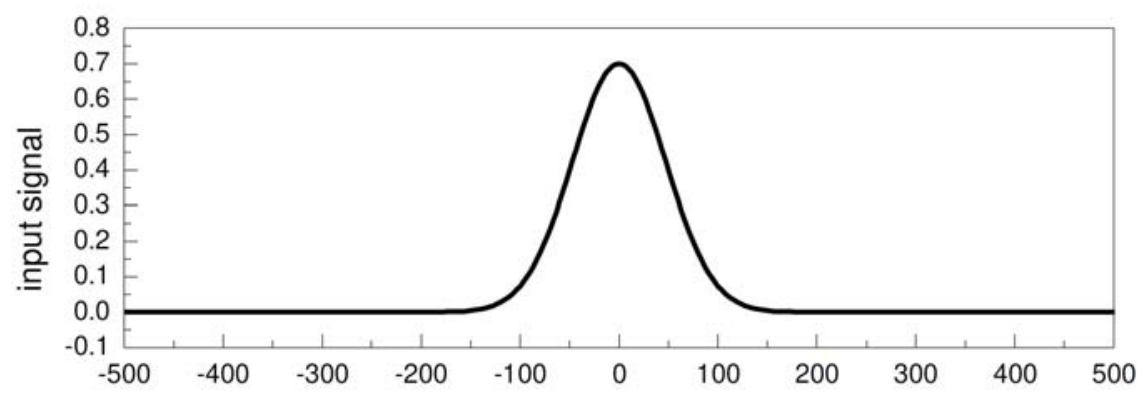

(a)

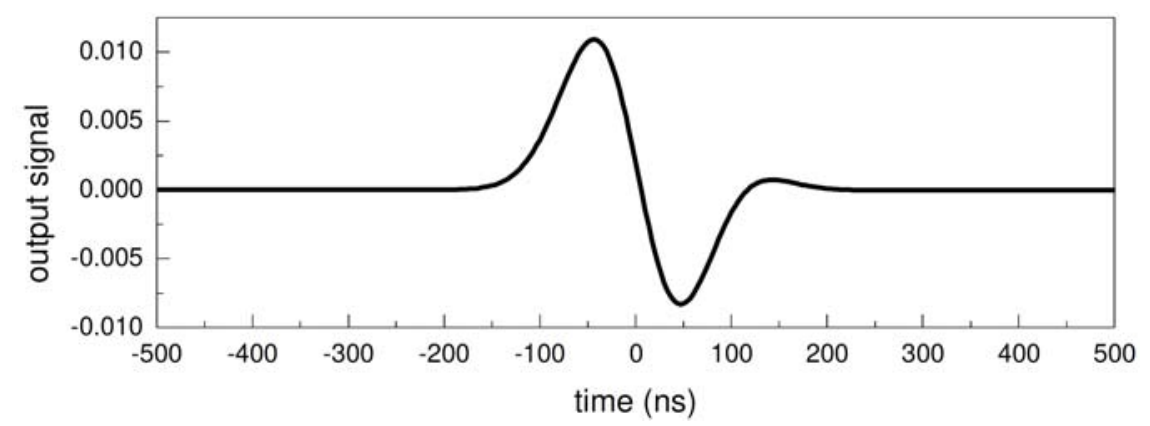

(b)

Figure 3. Time evolutions of input pulses in the system of EITT configuration with (a) waveforms of input pulses with Gauss-shape the duration of Gauss pulse $\tau=53.43 \mathrm{~ns}$ and (b) waveforms of output pulses. The used parameters are $N=1.2 \times 10^{10} \mathrm{~cm}^{-3}, \Omega_{p 2}=1.6 \gamma_{2}$, $\Omega_{+}=\Omega_{-}=6 \gamma \quad$ and $\quad \Omega_{p 1}=0.02 \gamma_{2}, \quad$ where $\quad \gamma=2 \pi \times 6.065 \mathrm{MHz}$; $\gamma_{2}=\Gamma_{41} / 2=2 \pi \times 0.97 \mathrm{MHz}$.

\section{Conclusion}

In conclusion, we demonstrate a scheme of all-optical transistor in hot standing-wave dressed atoms. Two optical blockades can be generated in each of the two types of structures of the system. The alloptical switching or in a temporal differentiator have been demonstrated to be created in the configurations of two-photon resonance and EITT, respectively. It is a potential device in optical logical circuits. 


\section{Author contributions}

Conceptualization, Xue Mei Su and Zhong Chang Zhuo; Software, Rui

Zhang; Formal analysis, Huifang Zhang; Writing-review and editing, Xue Mei Su.

Funding: This research was funded by National Scientific

Foundation China, grant number No.11174109.

\section{Acknowledgement}

In this section, the author acknowledged helpful discussion with Prof. Min Xiao of Physics Department of University of Arkansas, USA.

\section{References}

[1] F. Dell'Anno, S. D. Siena and F. Illuminati, Multiphoton quantum optics and quantum state engineering, Physics Reports 428(2-3) (2006), 53-168.

DOI: https://doi.org/10.1016/j.physrep.2006.01.004

[2] N. V. Vitanov, M. Fleischhauer, B. W. Shore and K. Bergmann, Coherent manipulation of atoms and molecules by sequential laser pulses, Advances in Atomic, Molecular, and Optical Physics 46 (2001), 55-190.

DOI: https://doi.org/10.1016/S1049-250X(01)80063-X

[3] T. C. Weinacht, J. Ahn and P. H. Bucksbaum, Controlling the shape of a quantum wavefunction, Nature 397(6716) (1999), 233-235.

DOI: https://doi.org/10.1038/16654

[4] Wenlan Chen, Kristin M. Beck, Robert Bücker, Michael Gullans, Mikhail D. Lukin, Haruka Tanji-Suzuki and Vladan Vuletic, All-optical switch and transistor gated by one stored photon, Science 341(6147) (2013), 768-770.

DOI: https://doi.org/10.1126/science.1238169

[5] Jzsef Fortagh and Claus Zimmermann, Magnetic microtraps for ultracold atoms, Reviews of Modern Physics 79(1) (2007), 235-289.

DOI: https://doi.org/10.1103/RevModPhys.79.235

[6] Serge Feneuille, Theoretical study of the resonances of a two level atom perturbed by a quasi-resonant radiation, Journal of Physics B: Atomic and Molecular Physics 7(15) (1974), 1981-1989.

DOI: https://doi.org/10.1088/0022-3700/7/15/006 
[7] L. Roso, R. Corbalan, G. Orriols, R. Vilaseca and E. Arimondo, Dressed-atom approach for probe spectroscopy in Doppler-broadened three-level systems with standing-wave saturator, Applied Physics B 31(2) (1983), 115-129.

DOI: https://doi.org/10.1007/BF00702607

[8] Haibin Wu, J. Gea-Banacloche and Min Xiao, Observation of intracavity electromagnetically induced transparency and polariton resonances in a dopplerbroadened Medium, Physical Review Letters 100(17) (2008); Article 173602.

DOI: https://doi.org/10.1103/PhysRevLett.100.173602

[9] M. A. Anton, Oscar G. Calderon, Sonia Melle, I. Gonzalo and F. Carreno, All-optical switching and storage in a four-level tripod-type atomic system, Optics Communications 268(1) (2006), 146-154.

DOI: https://doi.org/10.1016/j.optcom.2006.06.079

[10] S. E. Harris, Electromagnetically induced transparency, Physics Today 50(7) (1997), 36-42.

DOI: https://doi.org/10.1063/1.881806

[11] Min Xiao, Yong-Qing Li, Shao-Zheng Jin and Julio Gea-Banacloche, Measurement of dispersive properties of electromagnetically induced transparency in rubidium atoms, Physical Review Letters 74(5) (1995), 666-669.

DOI: https://doi.org/10.1103/PhysRevLett.74.666

[12] G. S. Agarwal and T. N. Dey, Ultraslow light in inhomogeneously broadened media, Physical Review A 73(4) (2006); Article 043809.

DOI: https://doi.org/10.1103/PhysRevA.73.043809

[13] M. G. Payne, L. Deng and K. J. Jiang, Limitations of steady state solutions to a two-state model of population oscillations and hole burning, Physical Review A 74(4) (2006); Article 043810.

DOI: https://doi.org/10.1103/PhysRevA.74.043810

[14] A. Andre and M. D. Lukin, Manipulating light pulses via dynamically controlled photonic band gap, Physical Review Letters 89(14) (2002); Article 143602.

DOI: https://doi.org/10.1103/PhysRevLett.89.143602

[15] Xue Mei Su, Jiteng Sheng and Min Xiao, Coupled atomic coherences induced by a standing wave, Optics Communications 318 (2014), 120-127.

DOI: https://doi.org/10.1016/j.optcom.2013.12.031

[16] Z. R. Chen, X. M. Su, J. B. Kim and Z. C. Zhuo, Observation of electromagnetically induced photonic band gaps in hot two-level atoms, Journal of Modern Optics 61(11) (2014), 898-903.

DOI: https://doi.org/10.1080/09500340.2014.914588 
[17] A. W. Brown and Min Xiao, All-optical switching and routing based on an electromagnetically induced absorption grating, Optics Letters 30(7) (2005), 699-701.

DOI: https://doi.org/10.1364/OL.30.000699

[18] M. Bajcsy, A. S. Zibrov and M. D. Lukin, Stationary pulses of light in an atomic medium, Nature 426(6967) (2003), 638-641.

DOI: https://doi.org/10.1038/nature02176

[19] F. Silva, J. Mompart, V. Ahufinger and R. Corbalan, Electromagnetically induced transparency with a standing-wave drive in the frequency up-conversion regime, Physical Review A 64(3) (2001); Article 033802.

DOI: https://doi.org/10.1103/PhysRevA.64.033802

[20] D. W. Li, L. Zhang, Z. C. Zhuo, J. B Kim and X. M. Su, Electromagnetically induced photonic bandgap in hot Cs atoms, Journal of the Optical Society of America B 27(4) (2010), 690-695.

DOI: https://doi.org/10.1364/JOSAB.27.000690

[21] Sungwon Kim, Zuo Wang, David J. Hagan, Eric W. Van Stryland, Andrey Kobyakov, Falk Lederer and Gaetano Assanto, Phase-insensitive all-optical transistors based on second order nonlinearities, IEEE Journal of Quantum Electronics 34(4) (1998), 666-672.

DOI: https://doi.org/10.1109/3.663446 


\section{Appendix A: Matrix $Q$}

Matrix $Q$ is expressed as:

$$
Q=\left(\begin{array}{ll}
A & B \\
C & D
\end{array}\right)
$$

here all the elements of matrix $A, B, C$, and $D$ are zero except the followings as:

$$
\begin{aligned}
& A(1,1)=-\widetilde{A}_{41} ; A(1,3)=A(5,7)=-i \grave{U}_{p 1} ; A(1,6)=\widetilde{A}_{21}-\widetilde{A}_{41} ; \\
& A(2,2)=-i\left(\ddot{A}_{p 1}-\ddot{A}_{c}\right)-\gamma_{21} ; A(2,3)=-i \grave{U}_{c} ; A(3,1)=A(7,5)=-i \grave{U}_{p 1} ; \\
& A(3,2)=A(7,6)=-i \grave{U}_{c}^{*} ; A(3,3)=-i \ddot{A}_{p 1}-\gamma_{31} ; A(3,4)=-i \grave{U}_{p 2} ; \\
& A(4,3)=-i \grave{U}_{p 2}^{*} ; A(4,4)=-i\left(\ddot{A}_{p 1}+\ddot{A}_{p 2}\right)-\gamma_{41} ; A(5,5)=i\left(\ddot{A}_{p 1}-\ddot{A}_{c}\right)-\gamma_{21} ; \\
& A(6,1)=-\tilde{A} 42 ; A(6,6)=-(\tilde{A} 21+\tilde{A} 42) ; A(6,7)=-i \grave{U}_{c} ; \\
& \left.A(7,7)=-i \ddot{A}_{c}\right)-\gamma_{32} ; A(7,8)=-i \grave{U}_{p 2} ; A(8,7)=-i \grave{U}_{p 2}^{*} ; \\
& A(8,8)=-i\left(\ddot{A}_{c}+\ddot{A}_{p 2}\right)-\gamma_{42} ; B(1,1)=i \grave{U}_{p 1}^{*} ; B(1,3)=\widetilde{A}_{31}-\widetilde{A}_{41} ; \\
& B(2,2)=i \grave{U}_{p 1}^{*} ; B(3,3)=i \grave{U}_{p 1}^{*} ; B(4,4)=i \grave{U}_{p 1}^{*} ; B(5,1)=i \grave{U}_{c}^{*} ; B(6,2)=i \grave{U}_{p 1}^{*} ; \\
& B(6,3)=\tilde{A}_{32}-\tilde{A}_{42} ; B(7,3)=i \grave{U}_{p 1}^{*} ; B(8,4)=i \grave{U}_{p 1}^{*} ; C(1,1)=i \grave{U}_{p 1} ; \\
& C(1,5)=i \grave{U}_{c} ; C(2,2)=i \grave{U}_{p 1} ; C(2,6)=i \grave{U}_{c} ; C(3,1)=C(3,6)=-\widetilde{A}_{43} ; \\
& C(3,3)=i \grave{U}_{p 1} ; C(3,7)=i \grave{U}_{c} ; C(4,1)=C(4,6)=-i \grave{U}_{p 2}^{*} ; C(4,4)=i \grave{U}_{p 1} ; \\
& C(4,8)=i \grave{U}_{c} ; C(8,1)=C(8,6)=i \grave{U}_{p 2} ; D(1,1)=i \ddot{A}_{p 1}-\gamma_{31} ; \\
& D(1,3)=D(5,8)=-i \grave{U}_{p 1} ; D(1,5)=D(2,6)=D(3,8)=i \grave{U}_{p 2}^{*} ;
\end{aligned}
$$


$D(2,2)=i \ddot{A}_{c}-\gamma_{32} ; D(2,3)=D(6,7)=-i \grave{U}_{c} ; D(3,1)=D(7,5)=-i \grave{U}_{p 1}^{*} ;$

$D(3,2)=D(7,6)=-i \grave{U}_{c}^{*} ; D(3,3)=\widetilde{A}_{31}+\widetilde{A}_{32}+\widetilde{A}_{43} ; D(3,4)=-i \grave{U}_{p 2} ;$

$D(4,3)=-2 i \grave{U}_{p 2}^{*} ; D(4,4)=-i \ddot{A}_{p 2}-\gamma_{43} ; D(5,1)=D(6,2)=i \grave{U}_{p 2} ;$

$D(5,5)=i\left(\ddot{A}_{p 1}+\ddot{A}_{p 2}\right)-\gamma_{41} ; D(6,6)=i\left(\ddot{A}_{c}+\ddot{A}_{p 2}\right)-\gamma_{42} ; D(7,3)=2 i \grave{U}_{p 2} ;$

$D(7,7)=i \ddot{A}_{p 2}-\gamma_{43}$. 